\title{
Six-monthly appointment spacing for clinical visits as a model for retention in HIV Care in Conakry-Guinea: a cohort study
}

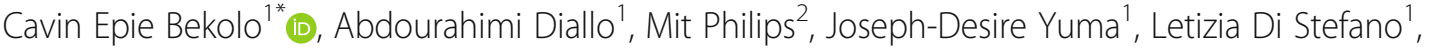
Stéphanie Drèze ${ }^{1}$, Jerome Mouton ${ }^{1}$, Youssouf Koita ${ }^{3}$ and Ousseni W. Tiomtore ${ }^{4}$

\begin{abstract}
Background: The outbreak of the Ebola virus disease (EVD) in 2014 led to massive dropouts in HIV care in Guinea. Meanwhile, Médecins Sans Frontières (MSF) was implementing a six-monthly appointment spacing approach adapted locally as Rendez-vous de Six Mois (R6M) with an objective to improve retention in care. We sought to evaluate this innovative model of ART delivery in circumstances where access to healthcare is restricted.

Methods: A retrospective cohort study in 2014 of the outcome of a group of stable patients (viral load $\leq 1000$ copies $/ \mu \mathrm{l}$ ) enrolled voluntarily in R6M compared with a group of stable patients continuing standard one to three monthly visits in Conakry. Log-rank test and Cox proportional hazards model were used to compare rates of attrition (deaths and defaulters) from care between the two groups. A linear regression analysis was used to describe the trend or pattern in the number of clinical visits over time.

Results: Included were 1957 adults of 15 years old and above of whom 1166 (59.6\%) were enrolled in the R6M group and 791 (40.4\%) in the standard care group. The proportion remaining in care at 18 months and beyond was 90\% in the R6M group; significantly higher than the 75\% observed in the control group $(p<0.0001)$. After adjusting for duration on ART and tuberculosis co-infection as covariates, the R6M strategy was associated with a $60 \%$ reduction in the rate of attrition from care compared with standard care (adjusted Hazard Ratio =0.40, 95\%Cl: 0.27-0.59, $p<0.001$ ). There was a negative secular trend in the number of monthly clinical visits for 24 months as the predicted caseload reduced on average by just below 50 visits per month $\left(\beta=-48.6, R^{2}=0.82, p<0.0001\right)$.

Conclusion: R6M was likely to reduce staff workload and to mitigate attrition from ART care for stable patients in Conakry despite restricted access to healthcare caused by the devastating EVD on the health system in Guinea. R6M could be rolled out as the model of care for stable patients where and when feasible as a strategy likely to improve retention in HIV care.
\end{abstract}

Keywords: ART delivery, Retention in HIV care, Ebola epidemic, Guinea

\section{Background}

In 2016, an estimated 19.5 million people living with Human Immunodeficiency Virus (HIV) were receiving antiretroviral therapy (ART) globally. This number is likely to increase exponentially in accordance with the current guidelines of the World Health Organisation (WHO) based on the 2030 vision to end the epidemic of Acquired Immunodeficiency Syndrome (AIDS) [1-5].

\footnotetext{
* Correspondence: msfocb-conakry-sida-epi@brussels.msf.org

${ }^{1}$ Médecins Sans Frontières, Conakry, Guinea

Full list of author information is available at the end of the article
}

The hopes for ending the AIDS epidemic may depend largely on the world's ability to provide ART to all HIVinfected patients but these hopes may be dashed if retention of people who have already initiated treatment is not optimal. Yet, HIV programmes especially in resource limited settings are faced with the daunting task of retaining the increasing number of people accessing ART $[3,6]$. Retention rates of $79.1 \%, 75.0 \%$ and $61.6 \%$ at 6,12 , and 24 months, respectively, have been reported in ART programmes in sub-Saharan Africa [7]. A 12-month retention of $79 \%$ was reported in five West African countries 
by The International Epidemiologic Databases to Evaluate AIDS (IeDEA) Collaboration [8]. For ART programmes to continue to expand while retaining people in care, WHO recommends a range of novel models of service delivery that, could be developed and implemented to respond to particular challenges in particular settings [3].

A number of health facility- and communitysupported models of ART delivery had been developed by Médecins Sans Frontières (MSF) in collaboration with local governments in sub-Saharan Africa to support on-going efforts to manage an ever-growing cohort of people on ART. These approaches include: appointment spacing for clinical and drug refill visits in Malawi, peer educator-led ART refill groups in South Africa, community ART distribution points in Democratic Republic of Congo (DRC) and patient-led community ART groups in Mozambique. These models have achieved high retention rates ranging from $89 \%$ at 12 months in DRC, $92 \%$ at 48 months in Mozambique, $94 \%$ at 36 months in Malawi, to $97 \%$ at 40 months in South Africa [9]. Other countries, including Uganda, South Africa and Zimbabwe, have taken a similar approach that allows for longer supplies of antiretroviral drugs in combination with spaced appointments $[9,10]$. These initiatives have so far been applied on stable patients in stable settings only.

In unstable or disaster settings however, the challenges of delivering routine healthcare are even more daunting as efforts are geared towards responding to the disruption caused by a given humanitarian emergency of concern [11]. Before the Ebola epidemic in Guinea, retention on ART at 12-months was approximately $75 \%$ [12]. When the outbreak of the Ebola virus disease (EVD) was declared in Guinea in 2014, a significant decrease in health service utilisation for HIV care was reported with rates of defaulting in ART care reaching $42 \%$ at the peak of the epidemic [13-15]. Prior to the EVD outbreak, MSF had begun piloting a six-monthly appointment for clinic and drug refill adapted locally as Rendez-vous de Six Mois (R6M) for stable HIV patients receiving ART, as a decongestion scheme to relieve pressure on its overstretched referral centre of Matam in Conakry and to improve retention in care. During the Ebola epidemic, the strategy was further deployed with an additional objective to reduce the risk of contracting EVD by reducing frequent contacts with the healthcare facility. We aimed to report this approach implemented during the EVD outbreak when access to healthcare was restricted.

\section{Methods}

A retrospective cohort study to compare attrition from R6M care relative to standard care after a follow up period of 24 months.

\section{Setting}

The HIV epidemic in Guinea remains largely overlooked by the rest of the world due to its low overall prevalence of $1.6 \%$, with approximately only one quarter of people living with HIV (approximately 120,000 in 2014) accessing antiretroviral treatment $[12,16]$. MSF has been providing HIV and tuberculosis (TB) services since the start of ART in 2003. In collaboration with the Ministry of Health, MSF provided support to over 7639 HIV patients in 2016 (24\% of the national ART cohort) through a decentralised approach in six health centres across the capital city, as well as at an outpatient clinic in Matam district [17]. In 2014, MSF was at the forefront of the Ebola response in West Africa where MSF teams treated 3804 patients in Guinea [18]. The Ebola outbreak had strongly affected Guinea's ability to provide HIV/AIDS services resulting to poor programmatic outcomes $[19,20]$.

\section{The R6M model of care}

R6M is a six-monthly appointment (SMA) model of ART care delivery for stable patients developed by MSF and implemented initially by The Chiradzulu HIV program in rural Malawi in 2008 with the objective to reduce patient waiting time and heath staff workload. Stable adults $(>95 \%$ adherence, current CD4 $\geq 300$ cells $/ \mu \mathrm{l}$ ), not pregnant, on first-line ART for more than a year and not presenting drug intolerance, tuberculosis or Kaposi's Sarcoma, were scheduled for clinical 6-monthly appointments by nurses and every 3 months for drug refill instead of every 1-2 months for patients in regular ART care $[9,21]$.

In 2013, MSF began piloting R6M in Conakry with a similar objective and approach to that in Malawi but by using a viral load $\leq 1000$ copies/ $\mu$ linstead of CD4 counts) as the main criterion to define a stable patient because routine viral load monitoring was implemented in MSF supported centres in Guinea. Approximately, $72.5 \%$ of the 7250 patients on ART for at least 6 months had had a least one viral load assay in MSF supported centres in June 2016. Of these, $90.7 \%$ had achieved viral load suppression. Initial results from implementing R6M prior to the EVD were encouraging [17]. Following the outbreak of EVD in 2014, R6M was scaled up with an additional dual objective of improving retention on ART and reducing the risk of EVD transmission. Patients outside the capital city were provided with ARV drugs enough to cover a period of 6 months while those residing in Conakry were provided with ARVs for 3 months by pharmacy attendants (pharmacy-only visits) and were seen by clinicians every 6 months. Exceptions to the rule were those who developed acute problems that compelled them to return earlier to seek medical attention. 


\section{Study design}

A retrospective cohort study of the outcome of a selfselected group of stable patients enrolled into the R6M care compared with a group of stable patients continuing standard care (the control group) at The Matam outpatient clinic supported by MSF in Conakry. Included in the R6M group were patients aged 15 years and above with a current viral load $\leq 1000$ copies/ $\mu$ l, non-pregnant and with no opportunistic infection (OI) between the 1st January 2014 and 31st December 2014. Included in the control group were patients of age 15 years and above with viral load $\leq 1000$ copies/ $\mu$, non-pregnant and with no current OI but continuing the routine 1-3 monthly follow-up visits during the same period. All patients eligible for this retrospective records review were included in the study. The choice to be included in either group was voluntary after adequate information had been provided by clinicians as in normal practice. Participants were free to quit or change groups at any time with support from their clinicians. Other baseline characteristics of the study groups were collected for comparability. Patients who were transferred out were excluded from the study irrespective of the group. The date of the last ART viral load defining eligibility was considered as the entry date into the cohort (time 0 ). The two groups were then observed throughout the duration of the EVD outbreak until 31st December 2015 (time of censoring) to determine their outcomes.

\section{Data collection}

We used routinely collected programme data that had been entered into the TIER.Net ${ }^{\circledR}$ 1.9.2.3 electronic medical record software developed by the University of Cape Town in South Africa. Abstracted for the purpose of the study where sociodemographic variables related to gender and age, clinical data pertaining to incident tuberculosis; current viral load titres and CD4 counts and their dates of measurements; date of ART initiation, current and prior ART regimens; the number of clinical visits; and their outcomes including the dates of ascertainment or censoring of the outcome. The dataset (Additional file 1) was then exported to Stata ${ }^{\oplus}$ software for statistical analysis.

\section{Statistical analysis}

Data analyses were performed using Stata ${ }^{\circledR}$ 14.2(StataCorp LP, TX77845, USA). The data set was checked for logical inconsistencies, illegal codes, omissions and improbabilities by tabulating, summarising, describing and plotting variables. Missing observations were excluded because they constituted a small random proportion.

Our main outcome of interest was attrition from care defined as a composite measure of the rate of occurrence of all-cause deaths and losses to follow-up (LTFU). A patient was classified as LTFU if there was no contact for 90 days or more after the last missed appointment for ARV refill [6]. MSF has a large team of lay workers who were used to provide continuous psychosocial support to patients in care and they were able through monthly home visits and/or telephone calls, to remain in contact with them. This effort in tracing patients was useful to encourage retention in care and to ascertain their outcome. Retention in care was used to indicate the proportion of patients alive and known to be still receiving ART at the time of the study [7]. The rate of attrition as a time to event variable was measured as the number of deaths and LTFU expressed over the length of time in person-months. The number of clinical visits per month was a secondary outcome automatically generated by the data entry software TIER.Net ${ }^{\circledR}$.

The main explanatory variable of interest was the exposure to R6M or standard model of care following results of the last ART viral load measurement. Other putative variables examined for comparability between the two groups included: gender, age, viral load titre, type of and duration on ART, last CD4 count and occurrence of a clinical event.

Summary statistics were presented as proportions for categorical variables and as means [with standard deviations (SD)] for normal continuous variables or medians [with Interquartile Ranges (IQR)] for skewed continuous variables. Pearson chi-squared tests or Fisher exact tests for small samples were used where appropriate to assess for differences among categorical variables between the two groups. The student t-test was used to test for the mean difference between the groups for continuous variables. Kaplan-Meier survival curves were used to display the rate of attrition over the study period meanwhile the log-rank test was used to assess for differences in survival probabilities between the two groups. A linear regression analysis was used to describe the trend or pattern in the number of clinical visits over time. A univariable Cox regression model was set up to screen for factors associated with attrition. Crude hazard ratios (HR) and their 95\%CI were obtained. The $p$-values for hypotheses testing were calculated from likelihood ratio tests (LRT). Variables found to be associated at 5\% confidence level, with attrition were included in a multivariable Cox model. Backwards elimination based on $p$-value lower than 0.05 was used to retain variables independently associated with attrition. The survival curves were then adjusted according to these independent predictors of attrition. The corresponding adjusted hazard ratios (aHR), their 95\% confidence intervals and $p$-values in the final model were reported [22]. The proportionality hazard assumption over time was assessed graphically using Aalen plots. An "intention- to- treat principle" was applied given that some patients in the R6M group returned to standard care and vice versa [23]. 


\section{Results}

\section{Descriptive characteristics of the study groups}

Included in a staggered manner between January and December 2014 were 1957 eligible adults of whom 1166 (59.6\%) were in the R6M group and 791 (40.4\%) in the standard care group (Fig. 1). During censorship, 202 (17\%) participants in the R6M had returned to standard care while $230(29 \%)$ of those in the control group had moved onto the R6M group. The mean period at risk was 16 months per subject or a total analysis time at risk of 31,220 person-months.

Participants in the R6M group were on average about 2 years older than the counterparts in the control group ( $41.8 \pm 10.8$ vs. $40.1 \pm 10.9$ years, $p=0.0007)$. The R6M group had significantly greater proportions of participants currently on Tenofovir (TDF)/Lamivudine (3TC)/Efavirenz (EFV) regimen $(82.7 \%$ vs. $53.7 \%, p<0.001)$, with ART experience of at least 5 years $(52.2 \%$ vs. $46.1 \%, p=0.005)$; and with an undetectable viral load below 250 copies/ $\mu$ l (98.2\% vs. $93.7 \%, \mathrm{p}<0.001)$ but all had achieved viral load suppression at $\leq 1000$ copies/ $\mu \mathrm{l}$ to become eligible for the study. The two groups were comparable in terms of gender composition, risk of a new opportunistic infection (active tuberculosis) or levels of current follow-up CD4 counts (Table 1).

\section{Trends in attrition from care}

After a total observation period of 24 months, 116 participants had dropped out from care through death or LTFU of whom $57(4.9 \%)$ in the R6M group and 84 $(10.6 \%)$ in the control group $(p<0.001)$. The overall retention rates remained high during the EVD outbreak but were higher in the R6M group than in the control group (Fig. 2): $98.2 \%, 96.3 \%$ and $95.8 \%$ at 6,12 and 18 months respectively in the R6M group against $95.4 \%$, $91.9 \%$ and $90.8 \%$ at 6,12 and 18 months respectively in the control group $(p<0.0001)$.

Attrition rates also differed between subjects who became coinfected with tuberculosis during follow-up $(27.6 \%$ vs: $6.9 \%)$ and those who did not $(p<0.001)$. Similarly, persons with an ART experience of 5 years and above had a lower rate of attrition (4.0\% vs.10.4\%) than those on ART for less than 5 years $(p<0.001)$ as indicated in Table 2. Gender, age, current CD4 counts or viral loads were not associated with the outcome.

After adjusting for HIV/TB coinfection and the length of time on ART (Table 2), the proportion remaining in care at 18 months and beyond was $90 \%$ in the R6M group (Fig. 3) significantly higher than the $75 \%$ observed in the standard group $(p<0.0001)$. R6M was thus associated with a $60 \%$ reduction in the rate of attrition from

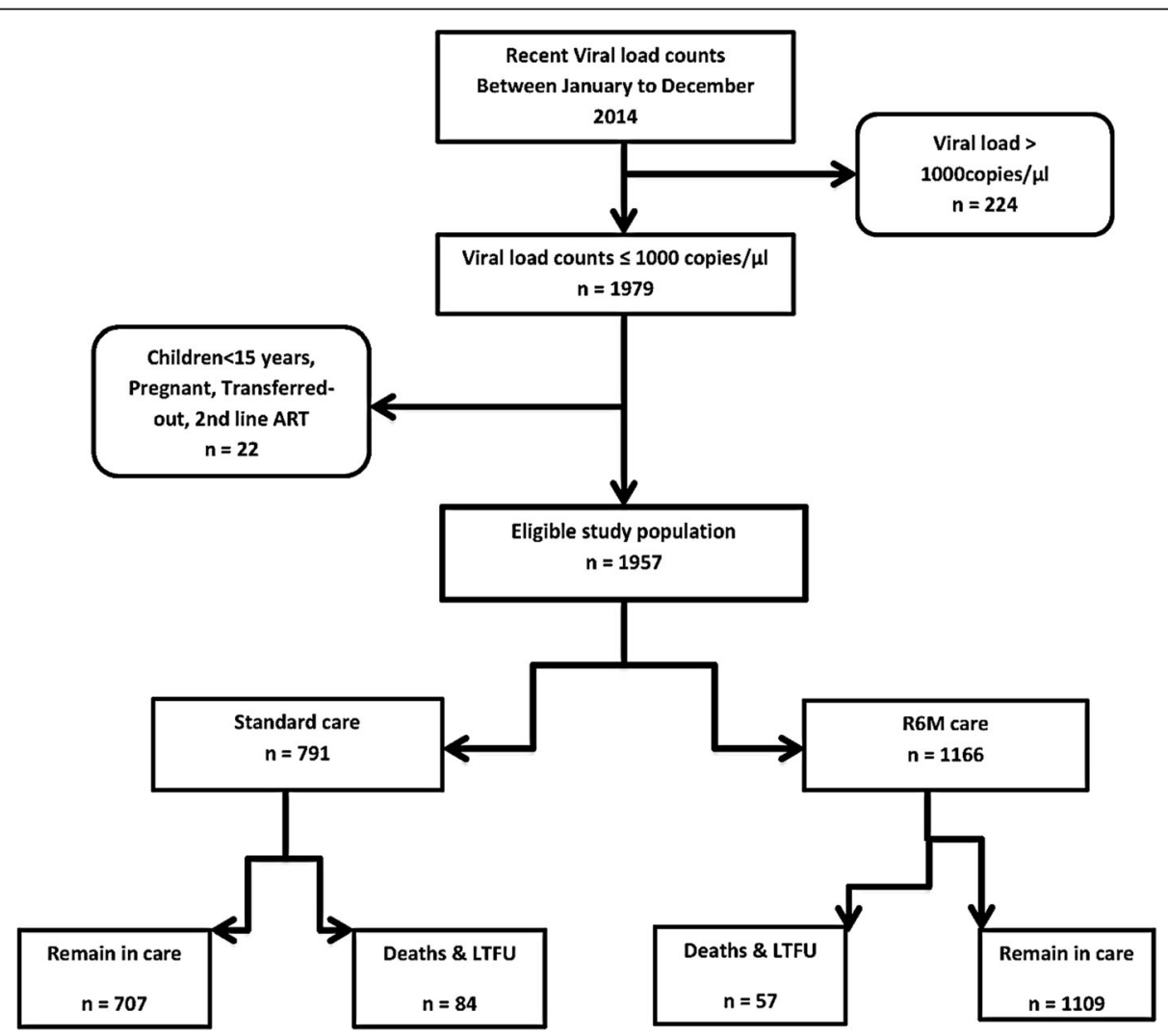

Fig. 1 Cohort flow chart 
Table 1 Baseline characteristics of the study groups

\begin{tabular}{|c|c|c|c|c|}
\hline \multicolumn{2}{|l|}{ Characteristics } & \multirow{2}{*}{$\begin{array}{l}\text { Standard care group } \\
(n=791)\end{array}$} & \multirow{2}{*}{$\begin{array}{l}\begin{array}{l}\text { R6M group } \\
(n=1166)\end{array} \\
41.8(10.8)\end{array}$} & \multirow{2}{*}{$\begin{array}{l}p \text {-value for difference } \\
\text { between the group } \\
0.0007\end{array}$} \\
\hline Age, mean (SD) years & & & & \\
\hline \multicolumn{5}{|l|}{ Gender, n (\%) } \\
\hline & Male & $229(28.9)$ & $383(32.8)$ & \\
\hline & Female & $562(71.0)$ & $783(67.2)$ & \\
\hline & Total & $791(100)$ & $1166(100)$ & 0.068 \\
\hline Current CD4 count, mec & & $497(261)$ & $494(243)$ & 0.769 \\
\hline \multicolumn{5}{|c|}{ Baseline Viral Load for eligibility in copies/ $\mu l, n(\%)$} \\
\hline & $<250$ & $741(93.7)$ & $1145(98.2)$ & \\
\hline & 250-1000 & $50(6.3)$ & $21(1.8)$ & \\
\hline & Total & $791(100)$ & $1166(100)$ & $<0.001$ \\
\hline \multicolumn{5}{|c|}{ TB status at any follow-up visit, $n$ (\%) } \\
\hline & Negative/Unknown & $778(98.4)$ & $1150(98.6)$ & \\
\hline & Positive & $13(1.6)$ & $16(1.4)$ & \\
\hline & Total & $791(100)$ & $1166(100)$ & 0.626 \\
\hline \multicolumn{5}{|c|}{ Duration on ART in months, $n(\%)$} \\
\hline & $<60$ & $419(53.9)$ & $557(47.8)$ & \\
\hline & $\geq 60$ & $358(46.1)$ & $609(52.2)$ & \\
\hline & Total & $777(100)$ & $1166(100)$ & 0.008 \\
\hline \multicolumn{5}{|c|}{ TDF-3TC-EFV regimen, $n$ (\%) } \\
\hline & No & $366(46.3)$ & $202(17.3)$ & \\
\hline & Yes & $425(53.7)$ & $963(82.7)$ & \\
\hline & Total & $791(100)$ & $1165(100)$ & $<0.001$ \\
\hline \multicolumn{5}{|l|}{ Outcome, n (\%) } \\
\hline & In care & $707(89.4)$ & $1109(95.1)$ & \\
\hline & Lost to Follow-up & $74(9.4)$ & $45(3.9)$ & \\
\hline & Dead & $10(1.3)$ & $12(1.0)$ & \\
\hline & Total & $791(100)$ & $1166(100)$ & $<0.001$ \\
\hline
\end{tabular}

care compared with standard care (adjusted Hazard Ratio $=0.40,95 \%$ CI: 0.27-0.59, $p<0.001)$.

\section{Trend in caseload over time}

Before the scale up of R6M in 2014, the number of clinical visits depicted a more or less cyclical or irregular pattern but thereafter, there was a negative secular trend. The number of clinical visits from January 2014 progressively decreased to about $50 \%$ by the end of December 2015 at the outpatient clinic of Matam when the uptake of R6M was about $55 \%$ of the patients enrolled on ART (Fig. 4). A linear regression model fitted on data from 2014 (Fig. 5) predicted that the number of clinic visits decreased on average by just below 50 every month during this period $\left(\beta=-48.6, \mathrm{R}^{2}=0.82, p<0.0001\right)$. Thus, in practice, $\mathrm{R} 6 \mathrm{M}$ as it was implemented, the caseload of clinicians could be estimated according to the following expression:

$$
\text { Caseload }=2477-49 \text { visits per month }
$$

\section{Discussion}

In this observational study, we compared the R6M approach relative to standard care on the outcomes of stable patients receiving ART during an Ebola epidemic that considerably reduced utilisation of HIV services in Guinea. The study indicated that R6M was likely to reduce attrition in care by $60 \%$ for patients who selfselected into the model. We also found that implementation of R6M was linked to a reduction in caseload by about half over a 24-month period at a rate of 50 clinical visits per month on average. 


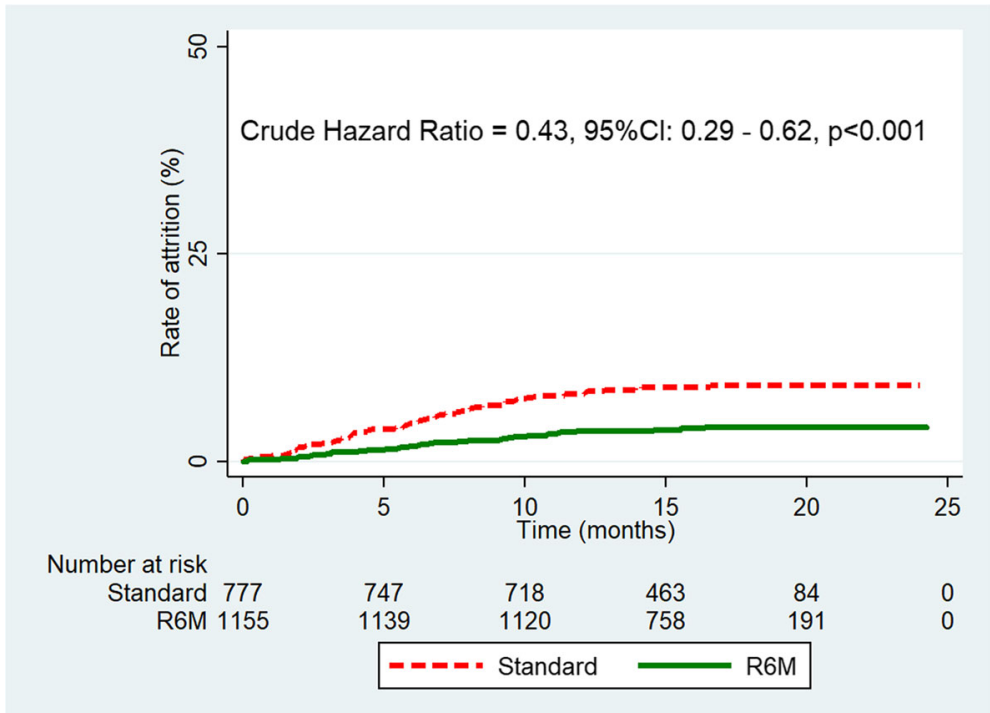

Fig. 2 Kaplan Meier curve of attrition from care

R6M is one of the several strategies for optimising long-term ART delivery developed and implemented by MSF in collaboration with Ministries of Health in subSaharan Africa. These models of care have already been integrated into $\mathrm{WHO}$ and UNAIDS guidelines and are gaining grounds in a growing number of countries across the continent in flexible ways according to context as "no one strategy fits all" [1, 9, 24]. None of these models has yet been tested in disaster settings. The context in Guinea was and remained one with a low HIV prevalence and a low ART coverage compounded by a devastating EVD that led to massive dropouts in care [12-14, 17]. Despite the disruption in healthcare delivery, stable patients receiving ART in general and those in R6M care in particular, demonstrated a tougher resilience by holding strong to their treatment. The success of a six-monthly appointment (SMA) on retention in care has been described in other settings at different time periods of follow-up. Findings from the Malawian cohort of Chiradzulu district that implemented a health facility driven model like R6M in Guinea had shown a retention rate of $96 \%$ at 24 months in 2014 [9]. In community driven models, SMA has equally demonstrated high retention rates: $89 \%$ at 12 months follow-up in DRC in 2012, 95.1\% after 20 months in rural Mozambique and 95.5\% in urban Mozambique in 2013; $92 \%$ at 15 months in the Thyolo model of Malawi and $100 \%$ at 5 months in the Roma model of Lesotho [24]. In contrast to R6M, and with the exception of DRC that has a low ART coverage (a characteristic common to West and Central Africa); results from studies listed above have been obtained from within Southern African countries with relatively stable and robust health systems to fight against HIV as indicated by their high ART coverage [17, 24, 25]. More so, their results were more descriptive than analytic from a methodological stand point. Results from our study have been derived from a comparative analysis in a disaster context where the

Table 2 Cox multiple regression model of factors associated with attrition from care $(n=1928)$

\begin{tabular}{|c|c|c|c|c|c|}
\hline Factor & Proportion of attrition (\%) & $\mathrm{HR}(95 \% \mathrm{Cl})$ & $p$-value & aHR (95\%Cl) & $p$-value \\
\hline \multicolumn{6}{|l|}{ Strategy } \\
\hline Standard & 10.6 & 1 & & 1 & \\
\hline R6M & 4.9 & $0.43(0.29-0.62)$ & $<0.001$ & $0.40(0.27-0.59)$ & $<0.001$ \\
\hline \multicolumn{6}{|l|}{ Incident tuberculosis } \\
\hline No & 6.9 & 1 & & 1 & \\
\hline Yes & 27.6 & $5.25(2.56-10.77)$ & $<0.001$ & $4.35(2.10-9.01)$ & 0.005 \\
\hline \multicolumn{6}{|c|}{ Duration on ART (months) } \\
\hline$<60$ & 10.4 & 1 & & 1 & \\
\hline$\geq 60$ & 4.0 & $0.42(0.28-0.62)$ & $<0.001$ & $0.47(0.31-0.71)$ & $<0.001$ \\
\hline
\end{tabular}




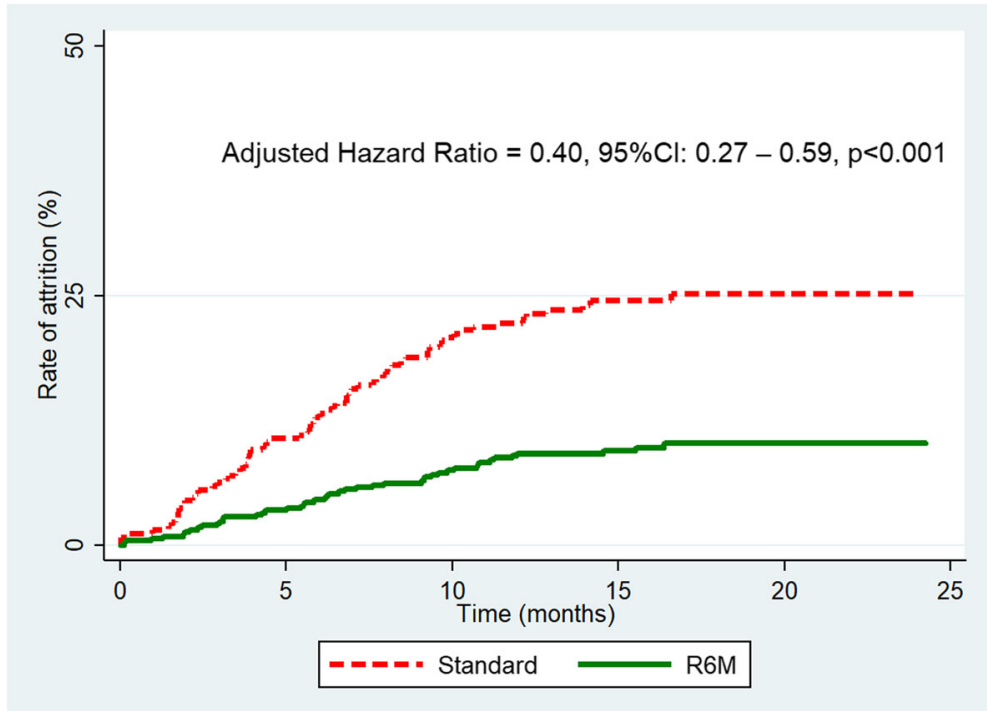

Fig. 3 Kaplan Meier curve of attrition in care adjusted for TB coinfection and treatment effect

HIV epidemic has been largely neglected [12, 17]. Despite differences in settings, uptake levels, implementation models and study designs, the evidences so far have been strong, coherent and consistent in favour of R6M or SMA as a strategy to improve retention in care. Therefore, ART programmes should be encouraged and supported to pick up or roll out to scale the implementation of SMA or any form of long appointment spacing and fast-track drug refill strategy. Some MSF-supported sites with access to routine viral load monitoring such as Malawi, South Africa and Zimbabwe are already moving to a once a year clinical visit with two to three monthly drug refills in between [24].

The National ART Programme in Guinea has endorsed R6M in principle but will have to meet a set of minimum requirements and critical enablers in order to overcome the challenges inherent to its implementation: A sufficient and flexible drug supply chain to permit ART dispensing of up to a 6 months' supply; a routine viral load testing not only to determine viral load suppression for R6M eligibility but also to detect treatment failure early enough so that patients could return to conventional care before dropping out of care like those who developed active TB in this study; a health information system to tract and monitor patients as they integrate $\mathrm{R} 6 \mathrm{M}$ or return to regular care and vice versa since the decision to participate is entirely voluntary and reversible at any time. The programme nonetheless has the potential to face these challenges: the state has been increasing its financial contribution to fight against HIV from $4 \%$ in 2011 to about $22 \%$ in 2014 [12, 26]; MSF and Solthis (Solidarité thérapeutique et initiatives contre

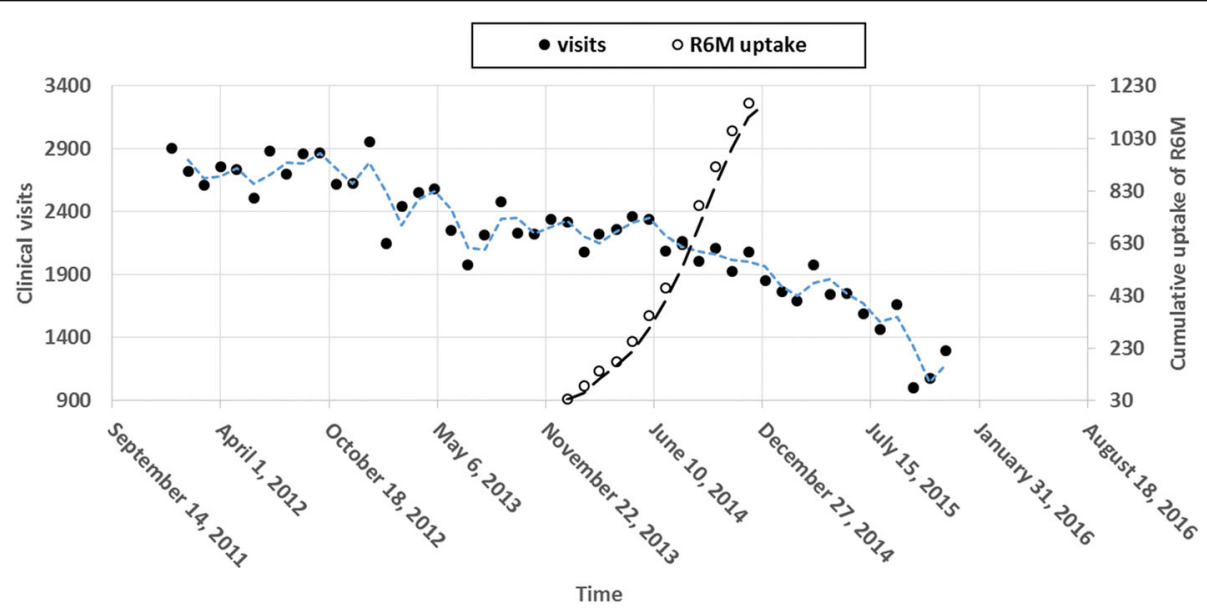

Fig. 4 Pattern of clinical visits and uptake of R6M over time 


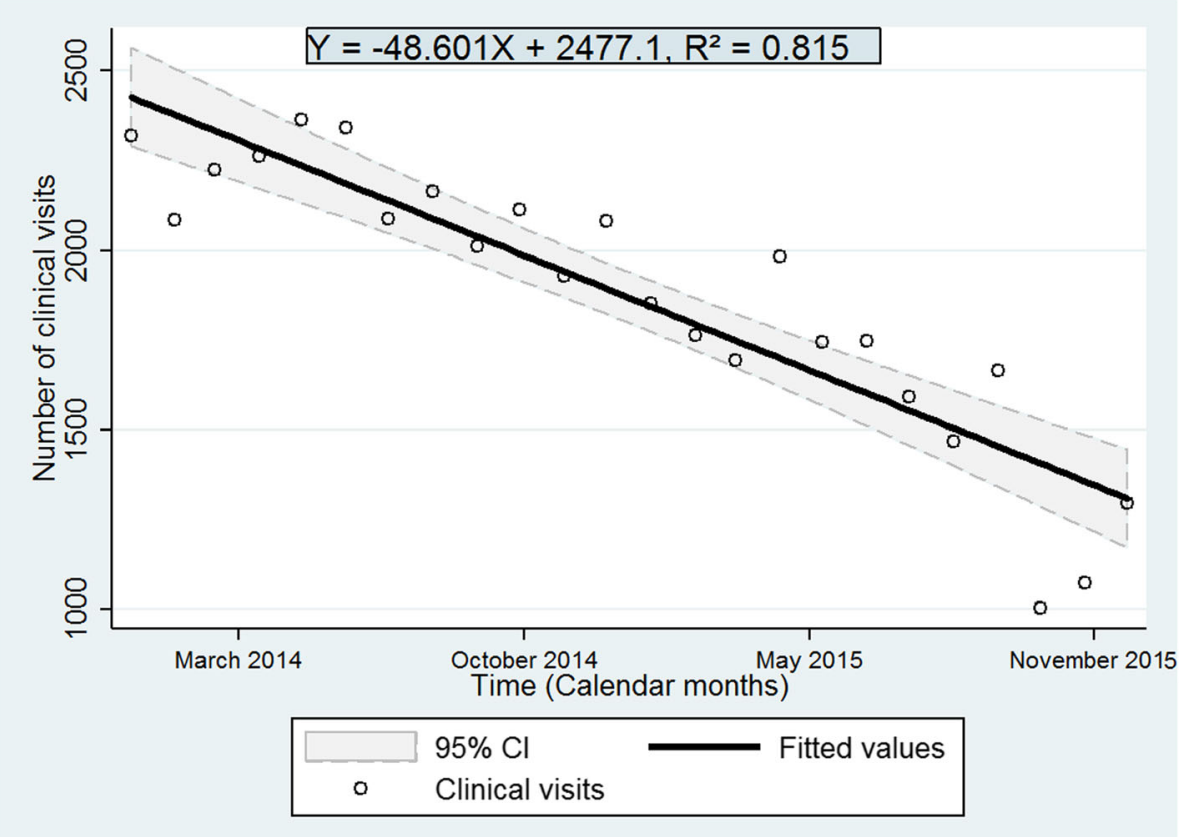

Fig. 5 Secular trend and prediction of caseload over time

le sida) via the Open Polyvalent Platforms (OPP-ERA) project funded by UNITAID are currently supporting the programme to ensure routine viral load monitoring of treatment response and in the implementation of monitoring and evaluation (M\&E) tools based on the electronic health information system. Yet, clinical and immunological monitoring of treatment response as well as paper-based M\&E tools already in use can be adapted to fit the R6M model. In rural Malawi for example, a CD4 $\geq 300$ cells/ $\mu$ in the absence of viral load was used as an eligibility criterion [9]. However, immunological and clinical criteria have poor sensitivity and specificity to detect treatment failure, particularly at higher CD4 cell counts [27]. The global response to the EVD in Guinea has also come with some investment for the country to rebuild a stronger health system for all programmes including the ART programme.

A reduction in the burden for healthcare workers and for patients has been the main goal for implementing R6M and community ART groups (CAG). This study has indicated that there had been a steady drop in the caseload for healthcare workers since instituting R6M at the Matam outpatient clinic. Though intuitive to link this reduction in workload to $\mathrm{R} 6 \mathrm{M}$, it is likely that the Ebola epidemic per se did contribute to a reduction in the frequency of contacts with the health facility as well as the decentralisation process integrated with R6M. Despite these intervening circumstances, we strongly believe that R6M can be singled out as the major contributor to the relief of the burden because the negative secular trend did not falter even when the epidemic had substantially receded in late 2015 and because efforts to decentralise patients have been less fruitful as patients do not voluntary accept to abandon the high quality of care offered at this clinic. We did not assess patient or healthcare worker satisfaction or benefits in this study, but given the experience from elsewhere, we can deduce that a reduction in the number of clinical visits would inevitably lead to a reduction in waiting times, travel costs and stigma; to improvement in individual patient empowerment to self-management, and of course to improvement in retention in care from the patient perspective $[9,24,28]$. Despite these benefits, some patients preferred to remain in conventional care because they perceived regular professional contact as more reassuring. From the healthcare service perspective, a reduction in the number of clinical visits would equally mean a reduction in the cost of healthcare delivery, a likely reduction in the risk of burn out syndrome and an improvement in the quality of care $[29,30]$. An economic evaluation as well as a patient/provider satisfaction surveys are recommended to validate these assumptions and to measure their real effects.

The study had other limitations. In routine care, patients "self-selected" conveniently or voluntarily into either treatment option. This obviously introduced selection bias but we thought that by choosing a 'control group' made of "stable" patients who were also eligible for R6M but opted to remain in conventional care after receiving adequate information including those related to knowledge and 
attitudes, from their clinicians, this could somehow reduce the bias. Collecting additional baseline data from both groups was done to identify and then control for any known differences between the two groups. Protocol violations were common because we observed that $17 \%$ of participants in the R6M group returned to conventional care while $23 \%$ of those in regular care opted for R6M during follow-up. This is a true reflection of routine care and of an observational study with a likely implication being a null effect of R6M but we believe this was taken care of by the "intent-to-treat" approach in the analysis. The period of observation of 24 months whereby only a maximum of four visits (against up to 24 visits for those in the control group) were possible for participants in the R6M group was rather short for a sufficient number of events to occur in this group. However, the R6M group was large enough to allow for accumulation of events in the group. Lack of randomisation in the design meant causality could not be assumed. The study did not directly measure the effect of R6M on the transmission of EVD but we can equally assume that if the number of clinician-patient contacts was reduced coupled with a reduction in waiting times and congestion in waiting rooms, the probability of an effective contact between a potential EVD contact or victim and a healthy person was also reduced [31]. Mathematical modelling could be recommended to simulate disease transmission dynamics in such a context.

\section{Conclusions}

R6M was likely to reduce staff workload and to mitigate attrition from care for stable patients in Conakry despite restricted access to healthcare caused by the devastating EVD on the health system in Guinea. R6M could be rolled out as the model of care for stable patients where and when feasible as a strategy to improve retention in HIV care.

\section{Additional file}

Additional file 1: The dataset (Additional file 1) was then exported to Stata ${ }^{\circledR}$ software for statistical analysis. (XLSX $199 \mathrm{~kb}$ )

\begin{abstract}
Abbreviations
3TC: lamivudine or 3-thiacytidine; aHR: adjusted Hazard Ratio; AIDS: Acquired Immunodeficiency Syndrome; ART: Antiretroviral Therapy; ARV: Antiretroviral (drug); CAG: community ART groups; CD4: Cluster designation 4 (of lymphocytes); EFV: Efavirenz; EVD: Ebola Virus Disease; HIV: Human Immunodeficiency Virus; HR: Hazard Ratio; leDEA: The International Epidemiologic Databases to Evaluate AIDS; IQR: Interquartile range; LTFU: Loss to follow up; MSF: Médecins Sans Frontières; Ol: Opportunistic infection; R6M: Rendez-vous de Six Mois (R6M); SD: Standard deviation; SMA: Six-monthly Appointment; TB: Tuberculosis; TDF: Tenofovir disoproxil fumarate; WHO: World Health Organisation
\end{abstract}

\section{Acknowledgements}

We are very grateful to all our patients and the national staff in Guinea for making the implementation of this intervention possible. We are also thankful to Steven Declerq and Luis Arias Moreno who at one time or the other supervised the implementation of this strategy; and to Boubacar
Aissata, Malick Doumbouya, John Milimono and Sekouna Sacko for helping with data collection. Jayne Webster and Joris van Loenhout provided the proofreading and editing support.

Funding

This study did not benefit from any specific funding.

Availability of data and materials

All data generated and analysed during this study are included in this manuscript and its Additional file 1.

\section{Authors' contributions}

Study conception and design: OWT, MP, CEB. Project implementation: JDY, LDS, SD, JM, YK. Data collection, cleaning and analysis: AD, LDS, CEB. Manuscript writing and revision: CEB, OWT, LDS, JDY, SD, JM, YK. All authors read and approved the final manuscript.

Ethics approval and consent to participate

The study was approved by the MSF Ethical Review Board. Individual consent was not necessary because the study used existing clinical data. All patient information was anonymised and de-identified prior to analysis.

\section{Consent for publication}

Not applicable

\section{Competing interests}

The authors declare that they have no competing interests.

\section{Publisher's Note}

Springer Nature remains neutral with regard to jurisdictional claims in published maps and institutional affiliations.

\section{Author details}

${ }^{1}$ Médecins Sans Frontières, Conakry, Guinea. ${ }^{2}$ Médecins Sans Frontières, Brussels, Belgium. ${ }^{3}$ National HIV/AIDS/STI Treatment \& Prevention Programme, Conakry, Guinea. ${ }^{4}$ Médecins Sans Frontières, Southern Africa Medical Unit (SAMU), Cape Town, South Africa.

Received: 30 January 2017 Accepted: 12 November 2017

Published online: 13 December 2017

\section{References}

1. WHO. Consolidated guidelines on the use of antiretroviral drugs for treating and preventing HIV infection: recommendations for a public health approach. Geneva: World Health Organization; 2013.

2. HIV/AIDS Fact sheet N³60 [http://www.who.int/mediacentre/factsheets/ fs360/en/].

3. WHO. March 2014 supplement to the 2013 consolidated guidelines on the use of antiretroviral drugs for treating and preventing HIV infection recommendations for a public health approach. Geneva: World Health Organization; 2014.

4. Joint United Nations Programme on HIV/AIDS (UNAIDS). 90-90-90 - An ambitious treatment target to help end the AIDS epidemic. In. Geneva; 2014

5. WHO. Consolidated guidelines on the use of antiretroviral drugs for treating and preventing HIV infection: recommendations for a public health approach. Geneva: World Health Organization; 2016.

6. WHO. Retention in HIV programmes: Defining the challenges and identifying solutions Meeting report (13-15 September 2011, Geneva, Switzerland): World Health Organization; 2012

7. Rosen S, Fox MP, Gill CJ. Patient retention in antiretroviral therapy programs in sub-Saharan Africa: a systematic review. PLoS Med. 2007:4(10):e298.

8. Ekouevi DK, Balestre E, Ba-Gomis FO, Eholie SP, Maiga M, Amani-Bosse C, Minga A, Messou E, Sow PS, Lewden C, et al. Low retention of HIV-infected patients on antiretroviral therapy in 11 clinical centres in West Africa. Trop Med Int Health. 2010;15(Suppl 1):34-42.

9. Bemelmans M, Baert S, Goemaere E, Wilkinson L, Vandendyck M, van Cutsem G, Silva C, Perry S, Szumilin E, Gerstenhaber R, et al. Community-supported models of care for people on HIV treatment in sub-Saharan Africa. Trop Med Int Health. 2014;19(8):968-77.

10. Nakiwogga-Muwanga A, Katabira E, Kiragga A, Kambugu A, Nakibuuka-Lubwama E, Manabe YC, Alamo ST, Colebunders R. Factors before enrolment are associated 
with being removed from a pharmacy-only refill Programme at a large urban HIV/AIDS clinic, Uganda. Int J STD AIDS. 2014;25(2):105-12.

11. Nickerson JW, Hatcher-Roberts J, Adams O, Attaran A, Tugwell P. Assessments of health services availability in humanitarian emergencies: a review of assessments in Haiti and Sudan using a health systems approach. Confl Health. 2015;9:20.

12. Comité National de Lutte contre le Sida (CNLS). Rapport National de la Riposte VIH/SIDA 2014 [Progres 2010-2014] en Guinee. In. Conakry; 2015.

13. Leuenberger D, Hebelamou J, Strahm S, De Rekeneire N, Balestre E, Wandeler G, Dabis F. Impact of the Ebola epidemic on general and HIV care in Macenta, Forest Guinea, 2014. AIDS. 2015;29(14):1883-7.

14. Ndawinz JD, Cisse M, Diallo MS, Sidibe CT, D'Ortenzio E. Prevention of HIV spread during the Ebola outbreak in Guinea. Lancet. 2015;385(9976):1393.

15. Brolin Ribacke KJ, Saulnier DD, Eriksson A, von Schreeb J. Effects of the West Africa Ebola virus disease on health-care utilization - a systematic review. Front Public Health. 2016:4:222.

16. Guinea HIV and AIDS estimates. 2014. [http://www.unaids.org/en/ regionscountries/countries/guinea]. Accessed 5 Dec 2017.

17. Médecins Sans Frontières (MSF). Out of Focus: How millions of people in West and Central Africa are being left out of the global HIV response. In. Brussels. 2016.

18. Ebola [http://www.msf.org/en/diseases/ebola]. Accessed 5 Dec 2017.

19. Pathmanathan I, Pevzner ES, Marston BJ, Hader SL, Dokubo EK. Insights from the Ebola response to address HIV and tuberculosis. Lancet Infect Dis. 2016; 16(3):276-8.

20. Kieny MP, Evans DB, Schmets G, Kadandale S. Health-system resilience: reflections on the Ebola crisis in western Africa. Bull World Health Organ. 2014;92(12):850.

21. McGuire M, Pedrono G, Mukhuna B, Huckabee M, Heinzelmann A, Szumilin E, Chikwaza O, Pujades-Rodriguez M. Optimizing patient monitoring after the first year of ART: three years of implementing 6-monthly clinical appointments in rural Malawi. In: 6th IAS conference on HIV pathogenesis, treatment and prevention: 2011. The International AIDS Society: Rome, Italy; 2011.

22. Vittinghoff EGD, Shiboski SC, CE MC. Regression methods in biostatistics. Boston, MA: Springer US; 2012

23. Montori VM, Guyatt GH. Intention-to-treat principle. CMAJ. 2001;165(10): 1339-41.

24. Médecins Sans Frontières (MSF). Reaching Closer to Home Progress implementing community-based and other adherence strategies supporting people on HIV treatment. Experiences from DRC, Lesotho, Malawi, Mozambique, South Africa \& Zimbabwe. In. Brussels. 2013.

25. Hill AM, Pozniak AN, Dauncey T, Levi J, Heath K, Essajee S, Elias CP. Countries with lower HIV prevalence have lower ARV coverage: UNAIDS 2015 database. In: Conference on retroviruses and opportunistic linfections 2016. Boston, Massachusetts: IAS-USA/CROI Foundation. p. 2016.

26. Comité National de Lutte contre le Sida (CNLS). Revue des progrès vers la réalisation des cibles de la déclaration 2011 de I'ONU sur le VIH et le Sida en Guinee. Rapport narratif 2014. In. Conakry. 2014: 36.

27. Rutherford GW AA, Easterbrook PJ, Horvath T, Vitoria M, Penazzato M, Doherty MC: Predicting treatment failure in adults and children on antiretroviral therapy: a systematic review of the performance characteristics of the 2010 WHO immunologic and clinical criteria for virologic failure. AIDS 2014, 28:161- 169

28. Campbell JL, Howie JG. Changes resulting from increasing appointment length: practical and theoretical issues. Br J Gen Pract. 1992;42(360):276-8.

29. Holden RJ, Scanlon MC, Patel NR, Kaushal R, Escoto KH, Brown RL, Alper SJ, Arnold JM, Shalaby TM, Murkowski K, et al. A human factors framework and study of the effect of nursing workload on patient safety and employee quality of working life. BMJ Qual Saf. 2011;20(1):15-24.

30. Portoghese I, Galletta M, Coppola RC, Finco G, Campagna M. Burnout and workload among health care workers: the moderating role of job control. Saf Health Work. 2014;5(3):152-7.

31. Brainard J, Hooper L, Pond K, Edmunds K, Hunter PR. Risk factors for transmission of Ebola or Marburg virus disease: a systematic review and meta-analysis. Int J Epidemiol. 2016;45(1):102-16.

\section{Submit your next manuscript to BioMed Central and we will help you at every step:}

- We accept pre-submission inquiries

- Our selector tool helps you to find the most relevant journal

- We provide round the clock customer support

- Convenient online submission

- Thorough peer review

- Inclusion in PubMed and all major indexing services

- Maximum visibility for your research

Submit your manuscript at www.biomedcentral.com/submit
Biomed Central 